\title{
Delirium-Prevention Programs in German and Austrian Hospitals - Views on Goals, Barriers, Facilitators and Implementation Procedures: A Qualitative Telephone Study Programme zur Delirprävention in deutschsprachigen Krankenhäusern: eine qualitative, telefonische Befragung zu den Zielstellungen, Barrieren, Förderbedingungen und Umsetzungsschritten in der Implementationsphase
}

\author{
Markus Wübbeler ${ }^{1 *}$, Sandra Bachmann', Julia Bringemeier ${ }^{2}$ \\ ${ }^{1}$ Hochschule für Gesundheit, Bochum, Department für \\ Pflegewissenschaft, 44801 Bochum, Germany \\ *markus.wuebbeler@hs-gesundheit.de \\ 2Evangelisches Klinikum Bethel, 33617 Bielefeld, Germany
}

Received 30 August 2018, accepted 21 January 2019

\begin{abstract}
Objectives: Analysis of barriers, facilitators and concepts to implement delirium prevention programs in German and Austrian hospitals.

Materials and Methods: Qualitative, semi-structured expert interviews with 9 leaders of delirium prevention programs in Germany and Austria.

Results: Leaders described delirium incidence reduction, higher standards in hospital care for older patients, and improvement of employee satisfaction as goals for their delirium prevention pro-gram. Barriers were described with fragmented hospital care structures - regarding sections and professional background of the staff - and lack of financial resources. Facilitators were named with acknowledging delirium prevention successes of known community leaders and building interest groups and networks. For the implementation of their delirium prevention program, hospitals used working groups and networks to benefit from outside perspectives on their implementation process.

Conclusion: Although delirium prevention is associated with cost savings in hospital procedures, program leaders described a lack of financial resources to implement delirium prevention programs. To face the demographic shift in hospital care, it is vital to implement national health care funding for delirium prevention programs. Currently, hospital leaders described an undersupply of older patients with delirium in German and Austrian hospital care.
\end{abstract}

\begin{abstract}
Ziele: Darstellung der Zielsetzungen, Barrieren, Förderbedingungen und Umsetzungsschritte im Rahmen der Implementation von Delirpräventionsprogrammen in Deutschland und Österreich.

Methode: Qualitative, semi-strukturierte Experteninterviews mit 9 Krankenhausakteuren aus dem Bereich der Implementation von Delirpräventionsprogrammen in Deutschland und Österreich.

Ergebnisse: Ziele sind die Reduktion der Delirinzidenz, Verbesserung der Behandlungsqualität und Erhöhung der Mitarbeiterzufriedenheit, als Barrieren werden die sektoralisierten Krankenhausprozesse, Finanzierungslücken und die personelle Umsetzung benannt. Die öffentliche Wahrnehmung des Programmes, eine gezielte interne und externe Kommunikation sowie finanzielle Mittel für hauptamtliches Personal wurden als Förderelemente identifiziert. Wichtige Elemente zur Umsetzung sind u. a. interdisziplinäre Arbeitsgruppen und Netzwerke zur Generierung von Synergieeffekten.

Schlussfolgerung: Eine Anschubfinanzierung ist ein wichtiger Schlüssel für eine leitliniengerechte Delirprävention. Derzeit stehen die sektoralisierten Krankenhausprozesse einer umfassenden, patientenorientierten Delirprävention in der klinischen Routine entgegen. Um dies aufzubrechen, müssen Förderprogramme initiiert werden, damit eine Schließung dieser Versorgungslücke im deutschsprachigen Raum zeitnah gelingt.
\end{abstract}

\section{Keywords}

Delirium - Prevention - Finance - Implementation

\section{Keywords}

Delirium - Prävention - Finanzierung - Implementation 


\section{EINLEITUNG}

Krankenhausaufenthalte sind bei älteren Patienten mit einer Abnahme der Funktionsfähigkeit und dem Auftreten kognitiver Dysfunktionen assoziiert. Bis zu 53\% der über 65 Jahren entwickeln im Verlauf des Krankenhausaufenthaltes ein Delir (American Geriatrics Society, 2015).

Mit dem Auftreten eines Delirs erhöhen sich die Wahrscheinlichkeit einer dauerhaften Pflegebedürftigkeit, irreversibler Kognitionsstörungen und das Mortalitätsrisiko (Fong, Tulebaev, \& Inouye, 2009). Das Delir ist ein Verwirrtheitszustand mit akutem Beginn, fluktuierendem Verlauf sowie Störungen in den Bereichen Bewusstsein, Aufmerksamkeit und Wahrnehmung (Young, Murthy, Westby, Akunne, \& O'Mahony, 2010). Ein Delir kann durch einen einzelnen Faktor ausgelöst werden, häufiger ist es jedoch die Folge multipler Stressoren. Dazu gehören Mangelernährung, die ungewohnte Krankenhausumgebung, Polypharmazie und soziale Isolation. Studien schätzen die finanziellen Folgen des Delirs in den Vereinigten Staaten auf 152 Milliarden US-\$ jährlich (Inouye, Westendorp, \& Saczynski, 2014). Etwa 40\% der Delirfälle sind jedoch vermeidbar (Inouye et al., 1999). Das Hospital Elder Life Program (HELP) bietet hierzu ein systematisches Interventionsprogramm, auch die NICE Organisation empfiehlt zentrale Interventionen des HELP-Programmes in ihrer Leitlinie zur Delirprävention (Yue et al., 2014). Seit HELP im Jahr 1999 durch eine Publikation im New England Journal of Medicine einer breiteren Fachöffentlichkeit bekannt wurde, hat es sich im Bereich der Delirprävention zu einem Standardprogramm entwickelt (Inouye et al., 1999). Bislang setzen mehr als 200 Krankenhäuser weltweit, überwiegend in den USA, das Programm erfolgreich ein.

HELP besteht aus 6 Kerninterventionen in den Bereichen Orientierung, Kognition, Ernährung, Wahrnehmung, Mobilität und Schlaf, welche jeweils mit einem Screeningund Interventionsprotokoll hinterlegt sind. Auf Basis eines Aufnahme-Screenings werden potenzielle DelirRisiken (u. a. älter als 70, eigeschränkte Kognition) mithilfe standardisierter Assessmentinstrumente (u. a. Kognitions-Screening) bestimmt und darauf aufbauend wird ein Interventionsplan entwickelt. Die Interventionen reichen von Orientierungs- und Kommunikationshilfen (u. a. Datum, Uhrzeit, Hörverstärker) sowie einer gezielten Gesprächs- und Beschäftigungsförderung (u. a. seniorengerechte Spiele, Vorleseaktivitäten), bis hin zur Frühmobilisation des Patienten und Unterstützung bei der Nahrungsaufnahme.

Das Programm wird wesentlich von Freiwilligen (Ehrenamtliche, geförderte Freiwilligendienste) getragen. Die Freiwilligen implementieren einen
Hauptteil der Interventionen unter Supervision eines geriatrisch qualifizierten Programmkoordinators. Neben den Freiwilligen und dem Programmkoordinator besteht ein HELP-Team zusätzlich aus einem Arzt mit geriatrischer Zusatzqualifikation und einer geriatrisch qualifizierten Pflegefachkraft. Die damit verbundenen Personalkosten bewegen sich in Abhängigkeit zur Größe des Krankenhauses, respektive der in das Programm aufgenommenen Patienten, bei mehr als 50.000 Euro pro Jahr. Durch die Verhinderung einer delirassoziierten Pflegebedürftigkeit, inkl. der damit verbunden Pflegekosten, ist die Prävention eines Delirs jedoch mittel-/ langfristig mit einer Kostenersparnis verbunden (Rubin, Neal, Fenlon, Hassan, \& Inouye, 2011).

Obwohl die Zahl der älteren Patienten auch in deutschen Krankenhäusern deutlich zugenommen hat und Delir ein verbreitetes klinisches Problem darstellt, werden strukturierte Delirpräventionsprogramme, mit wenigen Ausnahmen, bislang nicht in der deutschsprachigen Krankenhauslandschaft eingesetzt (Singler \& Thomas, 2017). Es stellt sich daher die Frage, welche Zielstellungen, Barrieren, Förderbedingungen und Umsetzungsschritte in deutschsprachigen Krankenhäusern mit der Implementation systematischer Delirpräventionsprogramme assoziiert sind?

\section{METHODE}

\section{Design}

Zur Untersuchung der Fragestellung wurde ein qualitatives Studiendesign mit semi-strukturierten, telefonbasierten Experteninterviews gewählt.

\section{Sample und Rekrutierung}

Die vorliegende Untersuchung wurde mit leitenden Mitarbeitern aus Krankenhäusern in Deutschland und Österreich durchgeführt. Eischlusskriterien für die hierzu durchgeführten qualitativen, semistrukturierten Telefoninterviews waren a) Teil des HELP-Netzwerks über eine Online-Registrierung (www.hospitalelderlifeprogram.org) oder Hospitation an einem deutschsprachigen HELP-Standort, b) eine verantwortliche Rolle im Implementationsprozess eines Delir-Versorgungsprogrammes und c) Standort des Krankenhauses in Deutschland oder Österreich. Da Delirpräventionsprogramme in den USA zu substanziellen Teilen über Finanzmittelsurrogate wie Freistellungen und Ehrenamt unterhalten werden und wenig über den Umsetzungsstand von Delirpräventionsmaßnahmen in deutschsprachigen Krankenhäusern bekannt ist, wurde eine explorative Herangehensweise gewählt. Zum Studienzeitpunkt war 
nur ein offizieller HELP-Standort registriert. Daher konzentrierte sich die Teilnehmerauswahl auf Personen/ Standorte die über das HELP-Netzwerk Zugriff auf die offiziellen Implementationsmaterialien (Manuale, Protokolle) hatten, ein erfolgreicher Aufbau, sowie eine damit verbundene Zertifizierung als HELP-Standort, jedoch nicht zu verzeichnen war. Per E-Mail wurden die Teilnehmer kontaktiert und über den Hintergrund sowie den Zweck der Studie aufgeklärt. Nach Einholung des Einverständnisses wurden die Teilnehmer zum verabredeten Zeitpunkt telefonisch kontaktiert und die Telefoninterviews audiotechnisch aufgezeichnet.

\section{Datenerhebung}

Die Telefoninterviews wurden von einem assoziierten Mitarbeiter des HELP-Central-Teams mit Sitz in Boston (USA) durchgeführt. Interviewer und die interviewten Personen waren sich im Vorfeld nicht bekannt, die Interviewsprache war deutsch. Der Interviewer beschäftigte sich mit dem Thema der Übertragbarkeit und Förderung des HELP-Modells in Deutschland und hatte ein detailliertes Vorverständnis über die praktische Umsetzung des HELP-Programmes. Die Interviews wurden zwischen Mai und Juli 2016 durchgeführt. Da der Erfahrungshintergrund $\mathrm{zu}$ den konkreten Umsetzungsschritten des HELP-Programmes im Vorfeld unklar war, enthielt der Interviewleitfaden offene Fragen zur Stimulation des Gesprächsverlaufes.

- Welches Ziel verfolgen Sie mit dem Delirpräventionsprogramm?

- Können Sie mir die bisherigen Schritte zur Umsetzung des Programms beschreiben?

- Welche Barrieren sind Ihnen bei der Umsetzung des Programms begegnet?

- Was würde die Umsetzung des Delirpräventionsprogramms fördern?

\section{Datenauswertung}

Zur Transkription der Interviews wurde die T5 Software (Marburg, Deutschland) verwendet, personenbezogene Daten entsprechend anonymisiert. Aufgrund der technisch bedingten Qualitätsverluste im Rahmen telefonischer Interviews wurde auf eine Transkription erweiterter Interviewmerkmale verzichtet und ein reduzierter Transkriptionsschlüssel verwendet. Das gesamte Material wurde verschriftlicht und in die Auswertung einbezogen. Zur Auswertung wurde die qualitative Inhaltsanalyse verwendet (Bengtsson, 2016): 1. Codierung des Materials mit entsprechenden Schlüsselkonzepten, 2. Zusammenfassung der Codierungen im Abgleich mit dem Originalmaterial, 3.
Kategorisierung der Codierungen, 4. Zusammenfassung der Kategorien. Zur Analyse wurde MAXQDA 12 genutzt (Berlin, Deutschland). Die Inhaltsanalyse wurde vom interviewenden Wissenschaftler durchgeführt, zur Erhöhung der Auswertungsqualität wurden die Themen mit einem erfahrenen HELP-Koordinator diskutiert (Lincoln \& Guba, 1985).

\section{ERGEBNISSE}

\section{Stichprobe}

Insgesamt konnten 9 Interviews mit einer Gesamtlänge von 7,5 Stunden, bei einer durchschnittlichen Dauer von 50 Minuten pro Interview, aufgezeichnet werden. Den überwiegenden Teil der Interviewpartner stellten Mediziner $\quad(n=5), \quad$ zweithäufigste Berufsgruppe waren Psychologen $(n=3)$ sowie ein Gerontologe. Die Befragten schilderten ihre Erfahrungen aus insgesamt 6 Krankenhäusern, 5 davon haben ihren Standort in Deutschland. Insgesamt arbeiteten 5 der 6 Krankenhäuser an eigenständigen Lösungen zum Aufbau eines Delirpräventionsprogramms und verwenden nur Teileaspekte des HELP-Programms. Ein Krankenhaus in der Stichprobe setzte sich zum Ziel, ein HELP-Programm zu implementieren (siehe Tabelle 1).

\section{Haupt- und Subkategorien}

\section{Hauptkategorie - Zielstellungen des \\ Delirpräventionsprogramms}

Im ersten Themenbereich schildern die interviewten Personen hohe Belastungen durch die Versorgung von älteren, deliranten Patienten im Krankenhaus. Der Bedarf für ein Delirpräventionskonzept ergibt sich demnach aus dem wachsenden Anteil älterer Patienten im Krankenhaus, dem damit verbundenen Anstieg von freiheitsentziehenden Maßnahmen (FEM) und dem funktionalen Abbau bei älteren Patienten. Aufgrund einer nicht für steigenden Anteil älterer Patienten angepassten Behandlungs- und Personalstruktur, kommt es im Krankenhaus vermehrt zu FEM (Fixierung, Sedativa) bei verwirrten, älteren Patienten.

Subkategorie - Freiheitsentziehende Maßnahmen im Kontext der Delirs:

$>$ [...] Eine nicht unerhebliche Quote von Patienten, die dann fixiert wurden, was eben ja menschenunwürdig ist in der Form und auch massive wiederum Risiken für den Patienten birgt und da gab es [...] Mitarbeiter, die sagten, das sind unhaltbare Zustände, da muss was passieren. <<(IP 7, 187-189) 
Tabelle 1: Eckdaten der befragten Krankenhäuser

\begin{tabular}{|c|c|c|c|c|c|c|}
\hline Kennzahlen & Nr. 1 & Nr. 2 & Nr. 3 & Nr. 4 & Nr. 5 & Nr. 6 \\
\hline Implementation HELP & 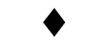 & - & - & - & - & - \\
\hline $\begin{array}{c}\text { Krankenhausspezifisches } \\
\text { Delirkonzept }\end{array}$ & & $\otimes$ & O & 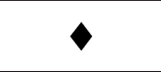 & $\Delta$ & O \\
\hline Anzahl Teilnehmer (Interviews) & 2 & 1 & 1 & 3 & 1 & 1 \\
\hline $\begin{array}{c}\text { Hauptamtliches Personal zur } \\
\text { Umsetzung }\end{array}$ & nein & nein & ja & ja & nein & ja \\
\hline Bettenanzahl & $<1000$ & $>2000$ & $<1000$ & $<1000$ & $>1000$ & $<1000$ \\
\hline Rechtsform & gGmbH & AöR & gGmbH & $\begin{array}{l}\text { Kommunaler } \\
\text { Träger }\end{array}$ & AöR & $\mathrm{GmbH}$ \\
\hline \multicolumn{7}{|l|}{ Finanzierung d. Programmes } \\
\hline Leistung Krankenhaus/Träger & - & - & ja & ja & - & ja \\
\hline Drittmittel & - & i. P. & ja & - & i. $P$. & ja \\
\hline Personalfreistellungen & ja & ja & ja & ja & ja & ja \\
\hline
\end{tabular}

Zielstellungen sind daher die Prävention des Delirs, Prävention kognitiver Defizite nach einem Krankenhausaufenthalt, Schaffung einer bedarfsgerechten Versorgung für ältere Krankenhauspatienten, die Reduktion von Wiedereinweisungsraten und die Senkung der Arbeitsbelastung. In diesem Zusammenhang steht zudem eine Erhöhung der Mitarbeiterzufriedenheit; das Personal wird dem selbst wahrgenommenen Versorgungsbedarf älterer Patienten nicht gerecht und zweifelt an der Erfüllung des gesetzten Behandlungsauftrages.

Subkategorie - Bedarfsgerechte Versorgung älterer Patienten:

>>Dass da also auch so eine Kultur von einem anderen Blick auf die alten Menschen entsteht und nicht nach Schema F das Auge, der Magen, das Knie gesehen wird. Und in dem Moment wo man merkt, dass da ein 80-jähriger multimorbider Mensch dranhängt, eher Hilflosigkeit und Passivität aufkommt. $<$ (IP 7 , 156-159)

Darüber hinaus wird auch eine Senkung der Krankenhauskosten erwartet, z. B. durch eine frühere Entlassung oder Vermeidung eines Drehtüreffektes.

\section{Hauptkategorie - Barrieren beim Aufbau eines Delirpräventionsprogramms}

Der Aufbau eines Delirpräventionsprogramms scheint durch die Krankenhausbedingungen erschwert. Demnach ist die moderne Krankenhausprozessstruktur vor allem auf einen hohen Patientendurchlauf ausgerichtet. Der dadurch entstehende Arbeitsdruck muss durch eine prozessoptimierte Taktung des Behandlungsablaufs kompensiert werden.

Subkategorie - Ausrichtung auf hohen Patientendurchlauf:

> Die größte Barriere im Akutkrankenhaus aus meiner Sicht (..) das Trimmen der Akutkrankenhäuser auf eine hochprofitable Gesundheitsfabrik (...) gar nicht an den Patientenbedürfnissen, sondern rein an der Mechanik der Machbarkeit ausgerichtet (...) und daher existieren heute meiner Ansicht nach nur noch Akutkrankenhäuser, die sich entsprechend angepasst haben. $<<($ IP 8, 521-523)

Die Implementation des Delirpräventionskonzepts erfordert nun eine Umstellung der Abläufe und hinterfragt zudem die bisherige Versorgungsqualität im Bereich Delir. Mit dem Ziel, die Berufsgruppen im Krankenhaus breiter an den Entscheidungs- und Behandlungsprozessen zu beteiligen, wird zudem das tradierte Hierarchiegefüge hinterfragt. Drüber hinaus konkurrieren Abteilungen miteinander um die knappen Ressourcen, sie versuchen ihre Interessen durchzusetzen und initiieren parallele Vorhaben, von denen sie sich einen Wettbewerbsvorteil versprechen.

Subkategorie - Konkurrierende Abteilungen im Krankenhaus:

>>Immer, wenn man interdisziplinär arbeitet, hat immer jeder seine eigenen Interessen vordergründig im Blick, das ist einfach so (...) z. B. die Zertifizierung der Klinik für Unfallchirurgie (...) Das war aber für 
die der Hauptkern, weil die sich ja zertifizieren lassen wollten. $<<($ IP 5, 337-340)

Die Nutzung von Freiwilligen im Krankenhaus wird unter ökonomischen Aspekten zwar begrüßt, jedoch als problematisch aufgefasst. Der Einsatz von Freiwilligen wird als unüblich beschrieben, eine ablehnende Haltung durch das Krankenhauspersonal gilt als wahrscheinlich.

Subkategorie - Der Einsatz von Freiwilligen im Krankenhaus:

> Diese Einbindung von Freiwilligen in den Krankenhausalltag, das gibt es hier eigentlich noch nicht (...) Wenn sowas vorschlägt, stößt man erst mal auf Irritationen (..) auf der Station, da gehören nicht irgendwelche Leute hin. $<<($ IP 5, 241-243)

Als zusätzliche Barriere gilt die Finanzierung des krankenhausspezifischen Delirkonzepts. Es wird davon ausgegangen, dass Aufwendungen und Personalkosten, die durch die Implementation des Konzeptes entstehen, nicht abrechnungsfähig sind. Daher müssen Krankenhäuser die Finanzierung aus Überschüssen und externen Mitteln ableiten.

Subkategorie - $\quad$ Finanzierung des Delirpräventionsprogramms:

>>Also man muss ganz klar sagen, wenn ein Krankenhaus was machen will, dann muss das Krankenhaus das nötige Geld für diese Maßnahmen an einer anderen Stelle erarbeiten, erwirtschaften, um es dann entsprechend ganz zielgerichtet einsetzen zu können.<<(IP 1, 282-284)

\section{Hauptkategorie - Förderbedingungen zum Aufbau eines Delirpräventionsprogramms}

Zum dritten Hauptthema schildern die Interviewten die wahrgenommenen Unterstützungsfaktoren bei der Implementation eines krankenhausspezifischen Delirkonzeptes. Hierzu gehören die öffentliche Wahrnehmung des Themas, gezielte interne Kommunikationsstrategien, eine Finanzierungsbasis für die Umsetzung und die Kooperation in Netzwerken.

Durch Auftritte gesundheitspolitischer Vertreter und die Anerkennung des Delirkonzeptes als Leuchtturmprojekt wird die Implementation auch im Innenverhältnis gestärkt. Die öffentliche Wahrnehmung und das durch die Medienpräsenz ausgelöste Feedback (u. a. Schilderungen von Betroffenen), bestärkt die Schlüsselakteure darin, ein wichtiges Problem zu adressieren. Neben regionalen und überregionalen Print- und Bildmedien wird die Abbildung des Delirkonzepts im Internet als wichtig erachtet.
Subkategorie - Öffentliche Wahrnehmung des Themas:

>>Einmal war sogar die Gesundheitsministerin da, um das Krankenhaus zu besuchen, und ich hatte da die Gelegenheit, das Ganze auch vorzustellen. Das hat dann später auch unserer Geschäftsführung nochmal klar gesagt, wie vorbildlich und förderungswürdig das ist. $<<($ IP 4, 229-232)

Für die Förderung des Delirkonzeptes ist eine adressatengerechte und gezielte interne Kommunikationsstrategie von Bedeutung. Je nach Krankenhausbereich gibt es Unterschiede in den Schlüsselaspekten, die es zu kommunizieren gilt. Für die Geschäftsführung ist das wirtschaftliche Risiko des Konzeptes entscheidend, weshalb sich die Akteure auf eine wirtschaftliche Bewertung des Konzepts vorbereiten sollten. Wichtige Eckpunkte sind die Gesamtkosten, Personalkosten und mögliche Erlöse, z. B. durch eine Verkürzung der Verweildauer. Grundsätzlich wird die Evidenzbasis des Konzeptes als wichtig erachtet, vor allem Führungskräfte erwarten eine wissenschaftliche Fundierung des Konzeptes.

\section{Subkategorie - Interne Datenbasis zur Bewertung des Konzepts: \\ >>Die Delirrate mehr als halbiert ( ) das war natürlich für den Geschäftsführer ein überzeugendes Argument, selbst wenn wir keine harten ökonomischen Zahlen erhoben haben. $<<($ IP 1, 132-134)}

Im Bereich der klinischen Bewertung ist es förderlich, auf Behandlungserfolge hinzuweisen, insbesondere bei Mitarbeitern, die über keine wissenschaftliche Ausbildung verfügen. Daher werden klinische Fallbesprechungen als förderlich beschrieben.

\section{Subkategorie - Klinische Fallbesprechungen zur Bewertung des Konzeptes: \\ >>Also da können die Kollegen ganz viel von profitieren, ich musste sie auch natürlich da ein bisschen hinlenken, die Erfolge auch wahrzunehmen (...) Dass sie also erste Erfolge der Intervention für sich erleben konnten. $<<$ (IP 8, 167-168)}

Die Befragten beschreiben Investitionsmittel als wichtigen Förderimpuls zur Implementation des Delirkonzepts. Drittmittel sind nicht nur für Projektinvestitionen wichtig, sie stellen auch einen notwendigen Aufbauimpuls dar. Mit den Geldern erhält das Delirkonzept einen Handlungsspielraum für die Anstellung hauptamtlicher Projektmitarbeiter, Materialinvestitionen und die finanzielle Aufwertung der Freiwilligenarbeit (z. B. Sozialversicherungsbeiträge). 
Insbesondere Krankenhausträger mit gemeinnütziger Orientierung betrachten das Delirkonzept mitunter als gute Reinvestition für erwirtschaftete Gewinne.

Subkategorie - Bedeutung hauptamtlicher Mitarbeiter: >>Aber was ich sagen kann, ist dieser hauptamtlicher Mitarbeiter, das ist eine ganz wichtige Größe das es eben nicht nur alle Akteure versuchen das nebenher zu machen. $<<$ (IP 7, 61-62)

>>Aber wir hoffen halt (...) dass wir Gelder ausmachen können. Also irgendwelches Seed Money erstmal (...) die es uns ermöglichen das einzurichten, mal laufen zu lassen und dann zu evaluieren. $<<$ (IP 2, 147-149)

Als unterstützend gilt zudem die Zusammenarbeit in Netzwerken. Sie ermöglichen eine Reflexion des eigenen Konzepts und fördern die Zusammenarbeit bei der Entwicklung neuer Maßnahmen. Hierzu gehören Interventionen, Schulungsangebote und strategische Erwägungen (z. B. Expansion des Konzeptes).

Subkategorie - Unterstützungsstruktur Netzwerk: $>$ Wir arbeiten ja auch mit dem ambulanten Demenznetzwerk eng zusammen (...) Also da ist in den zehn Jahren extrem viel entstanden. $<$ (IP 4, 76-78)

Hauptkategorie - Umsetzungsschritte zum Aufbau des Delirpräventionsprogramms

Der Aufbau des Delirpräventionsprogramms wird über die Bildung von interdisziplinären und fachabteilungsübergreifenden Arbeitsgruppen initiiert, beteiligte Berufsgruppen sind u. a. Pflegefachkräfte, Ärzte, Psychologen und Physiotherapeuten aus den mittleren und unteren Führungsebenen des Krankenhauses.

Subkategorie - Bildung einer Arbeitsgruppe:

$>>(\ldots)$ eine Arbeitsgruppe und die war, also es gab zwei sogar, es gab eine interdisziplinäre [Arbeitsgruppe] wo auch der (anonymisiert) drin ist und es gab eine in der Pflege. In der Regel waren diese Treffen einmal monatlich (...) immer direkt nach der Mittagsübergabe.<<(IP 9, 700-703)

Ergänzt wird diese Implementationsstruktur durch ein externes Netzwerk, welches mit einem unterschiedlichen Formalisierungsgrad unterhalten wird. Der Formalisierungsgrad reicht von unregelmäßigen Kontakten bis zu einer eigenständigen Rechtsstruktur. Die einrichtungsspezifischen Delirpräventionskonzepte setzen schließlich auf verschiedene Maßnahmen, die mit einer Delirprävention in Verbindung gebracht werden. Hierzu gehören Maßnahmen in den Bereichen der Krankenhausumgebungsgestaltung (u. a. Beleuchtung, Orientierungshilfen, Spezialbetten zur Sturzrisikoreduktion, Milieukonzept, spezialisierter DelirVersorgungsbereich), geriatrische Konsile (kollegiale und interdisziplinäre Fachberatung), Überleitungsmaßnahmen (Internes Überleitungsmanagement, Case Management), prä- und postoperative Begleitung der Risikopatienten (Bestimmung von Risikofaktoren, Patientenund Angehörigenberatung, Bewegungsübungen, OP-Begleitung), seniorengerechte Ernährung (Flüssigkeitshaushalt, Nährstoffgehalt, Textur) sowie Kommunikations-/Therapietechniken (Kommunikationstafeln, kognitive Stimulation).

\section{Subkategorie - Umgebungsgestaltung zur Delirprävention}

>>Wir haben zwei Intensivzimmer so eingerichtet, dass sie nicht mehr aussehen wir Intensivzimmer, wo die Patienten nach der OP hinkommen. Wo es entsprechende Beleuchtungen gibt die sich verändern lassen (...) ganz helles Licht, aber auch verschiedene Wellenlängen. Da ist Ruhe, da piepst nichts(...) das sieht eher aus wie so ein ja ein gepflegtes Schlafzimmer und nicht wie so eine übliche Intensivstation. $<<$ (IP 2, 21-27)

Das Krankenhaus, welches sich der Implementation des HELP-Programms widmet, orientiert sich an den standardisierten Interventionen und Protokollen der HELP-Manuale und verzichtet auf eine Ausweitung oder Neuinterpretation der Maßnahmen.

\section{DISKUSSION}

Die durchgeführte Studie hatte zum Ziel, die Zielstellungen, Barrieren, Förderbedingungen und Umsetzungsschritte im Bereich der Implementation von Delirpräventionsprogrammen in deutschsprachigen Krankenhäusern $\mathrm{zu}$ beschreiben. Im Rahmen der hierzu durchgeführten Telefoninterviews wird deutlich, dass die Verantwortlichen vor umfangreichen Herausforderungen stehen. Zentrale Probleme bestehen bei der bedarfsgerechten Versorgung älterer Patienten. Insbesondere hyperaktive Delirformen werden häufig nicht leitliniengerecht versorgt, Patienten durch medikamentöse und nicht-medikamentöse Maßnahmen ruhiggestellt. Die hierfür beschriebenen Ursachen sind die gestiegene Anzahl älterer Patienten und nicht-angepasste Behandlungsprozesse. Anvisierte Zielestellungen sind daher eine Reduktion der Delirinzidenz, die 
Verbesserung der Behandlungsqualität und Erhöhung der Mitarbeiterzufriedenheit. Die in diesem Zusammenhang beschriebenen Barrieren konzentrierten sich vor allem auf eine sektoralisierte Krankenhausstruktur, die als Folge einer Effizienzsteigerung im Krankenhauswesen beschrieben wird. Um das gestiegene Arbeitstempo verarbeiten $\mathrm{zu}$ können, haben die Abteilungen und Berufsgruppen ein arbeitsteiliges Prinzip implementiert, welches einer kooperativen und interdisziplinär ausgerichteten Delirprävention entgegensteht. Bei den beschriebenen Förderelementen zum Aufbau eines Delirpräventionsprogramms handelt es sich um die öffentliche Wahrnehmung, z.B. durch gesundheitspolitische Vertreter, eine gezielte interne und externe Kommunikation, Fallbesprechungen zur gezielten Betrachtung von Behandlungserfolgen sowie die finanzielle Förderung zur Anstellung/Freistellung von hauptamtlichen Mitarbeitern. Für den Aufbau der Delirprävention initiieren die Verantwortlichen schließlich interdisziplinäre Arbeitsgruppen, suchen externe Kontakte über den Aufbau von Netzwerken und verwenden eine Kombination verschiedener Therapiekonzepte u. a. zur Umgebungsgestaltung, präund postoperativer Versorgung sowie Kommunikationsund Therapietechniken.

Die Analyse zeigt, dass Krankenhäuser in Deutschland und Österreich, trotz einer schwierigen Finanzierungslage, an der Implementation und Entwicklung von Delir-Versorgungsprogrammen arbeiten. $\mathrm{Da}$ die leitliniengerechte Prävention und Versorgung des Delirs auf mehreren Interventionskomponenten basiert, stehen die befragten Akteure hier vor der Aufgabe, umfangreiche Komplexinterventionen in die klinische Praxis $\mathrm{zu}$ implementieren (National Clinical Guideline, 2010). Die damit verbundenen Prozesse sind ressourcenaufwendig, für eine erfolgreiche Implementation ist daher eine Grundfinanzierung erforderlich. Aus den Daten zur Begleitforschung des HELP-Programms wird ersichtlich, dass die Grundfinanzierung solcher DelirVersorgungsprogramme nicht ausschließlich ein nationales Problem ist. Krankenhäuser aus dem USamerikanischen Raum, in dem das HELP-Programm überwiegend Anwendung findet, berichten ebenfalls über Schwierigkeiten, die notwendige Grundausstattung (1,5-2,0 Vollzeitäquivalente) akquirieren zu können (Bradley, Webster, Baker, Schlesinger, \& Inouye, 2005). Der dringende Bedarf einer solchen Grundfinanzierung wurde auch von den Studienteilnehmern artikuliert.

Um eine Implementation einzuleiten, wird in den USA häufig auf Drittmittel- oder Entwicklungsfonds zurückgegriffen, eine Anschubfinanzierung ist dadurch nur für einen begrenzten Zeitraum sichergestellt. Darauf aufbauend werden zusätzlich Gelder aus dem Krankenhausbudget aufgebracht, um das Programm zu stützen, bzw. vollständig zu finanzieren. Entscheidend hierfür ist eine strukturierte Evaluation des Programms. Lassen sich die positiven Ergebnisse (u. a. Senkung der Delirrate, Erhöhung der Mitarbeiterzufriedenheit, Senkung der Widereinweisungsraten) empirisch zeigen, erhalten die Präventionsprogramme in vielen Fällen eine dauerhafte Finanzierung (Bradley et al., 2005). Vorrausetzung hierfür ist, das bereits früh an der Erhebung geeigneter Kennzahlen gearbeitet wird, um diese im Rahmen einer Evaluation darlegen zu können. Die Akteure im Krankenhaus stehen daher vor der Herausforderung, geeignete Daten zu sammeln. Dies gilt insbesondere für Programme, bei denen es sich um eigene Neuentwicklungen oder um eine Kombination verschiedener Präventionsansätze handelt. Im Vergleich zum HELP-Programm stehen krankenhausspezifische Konzepte hier meist nicht auf einem umfangreichen Evidenzkörper. Grade Komplexinterventionen stellen jedoch hohe Anforderungen an die Akteure, wenn es um ihre Evaluation geht (Campbell et al., 2000).

\section{LIMITATIONEN}

Bei der vorliegenden Studie sind einige Limitationen zu beachten:

Die Daten wurden nicht bis zum Erreichen der Sättigung erhoben. Die Datenauswertung wurde primär von dem interviewenden Wissenschaftler durchgeführt, zugunsten einer höheren Objektivität wäre eine Trennung bzw. Mehrfachauswertung empfehlenswert gewesen - dies konnte aus forschungsökonomischer Sicht nicht realisiert werden.DiedemografischenDatenderInterviewteilnehmer wurden im Forschungsprozess nicht dokumentiert. Da nur wenige Krankenhäuser an der Implementierung systematischer Delirpräventionsprogramme arbeiten, stand die Anonymisierung des Untersuchungsfeldes im Vordergrund.

\section{SCHLUSSFOLGERUNGEN}

Insgesamt lässt sich daher feststellen, dass die Anschubfinanzierung ein wichtiger Schlüssel für eine leitliniengerechte Delirprävention ist. Ist die Finanzierungslücke überwunden, stehen einer leitliniengerechten Prävention des Delirs insbesondere die sektoralisierten Krankenhausprozesse, mit einem Abteilungs- und Fächergruppenfokus, entgegen. Bestehende Initiativen sollten daher eine Vernetzung, sowie Dissemination ihrer Konzepte anstreben. Dieser Schritt kann die Ableitung eines einheitlichen Kriterienkatalogs für die Delirprävention im deutschsprachigen Raum fördern. Darüber hinaus sind die gemeinsame Entwicklung von Qualifikationsangeboten sowie die Nutzung von Effizienzgewinnen durch eine 
evidenzbasierte Implementationsstrategie zentrale Bausteine für eine breite Delirpräventionsstrategie im deutschsprachigen Raum. Im Zuge des demografischen Wandels ist eine Stärkung der Delirprävention unumgänglich, Förderprogramme müssen hier zukünftig die notwendigen Akzente setzen.

\section{DANKSAGUNG}

Wir danken allen Studienteilnehmern für die Mitwirkung an dieser Studie. Ausdrücklich danken wir der Robert-Bosch-Stiftung für die Projektförderung und Unterstützung des Vorhabens.

Literatur

Bengtsson, M. (2016). How to plan and perform a qualitative study using content analysis. NursingPlus Open, 2, 14. doi:10.1016/j. npls.2016.01.001

Bradley, E. H., Webster, T. R., Baker, D., Schlesinger, M., \& Inouye, S. K. (2005). After adoption: sustaining the innovation. A case study of disseminating the hospital elder life program. J Am Geriatr Soc, 53(9), 1455-1461. doi:10.1111/j.15325415.2005.53451.x

Campbell, M., Fitzpatrick, R., Haines, A., Kinmonth, A. L., Sandercock, P., Spiegelhalter, D., \& Tyrer, P. (2000). Framework for design and evaluation of complex interventions to improve health. Bmj, 321(7262), 694-696.

Fong, T. G., Tulebaev, S. R., \& Inouye, S. K. (2009). Delirium in elderly adults: diagnosis, prevention and treatment. Nat Rev Neurol, 5(4), 210-220. doi:10.1038/nrneurol.2009.24

Inouye, S. K., Bogardus, S. T., Jr., Charpentier, P. A., Leo-Summers, L., Acampora, D., Holford, T. R., \& Cooney, L. M., Jr. (1999). A multicomponent intervention to prevent delirium in hospitalized older patients. N Engl J Med, 340(9), 669-676. doi:10.1056/ nejm199903043400901

Inouye, S. K., Westendorp, R. G., \& Saczynski, J. S. (2014). Delirium in elderly people. Lancet, 383(9920), 911-922. doi:10.1016/ s0140-6736(13)60688-1

Lincoln, Y. S., \& Guba, E. G. (1985). Naturalistic inquiry. Beverly Hills, Calif.: Sage Publications.

National Clinical Guideline, C. (2010). National Institute for Health and Clinical Excellence: Guidance. In: Delirium: Diagnosis, Prevention and Management. London: Royal College of Physicians (UK)

National Clinical Guideline Centre - Acute and Chronic Conditions. Postoperative delirium in older adults: best practice statement from the American Geriatrics Society. (2015). J Am Coll Surg, 220(2), 136-148.e131. doi:10.1016/j.jamcollsurg.2014.10.019

Rubin, F. H., Neal, K., Fenlon, K., Hassan, S., \& Inouye, S. K. (2011). Sustainability and scalability of the hospital elder life program at a community hospital. J Am Geriatr Soc, 59(2), 359-365. doi:10.1111/j.1532-5415.2010.03243.x

Singler, K., \& Thomas, C. (2017). [HELP - Hospital Elder Life Program - multimodal delirium prevention in elderly patients]. Internist (Berl), 58(2), 125-131. doi:10.1007/s00108-016-0181-0
Young, J., Murthy, L., Westby, M., Akunne, A., \& O’Mahony, R. (2010). Diagnosis, prevention, and management of delirium: summary of NICE guidance. Bmj, 341, c3704. doi:10.1136/bmj. c3704

Yue, J., Tabloski, P., Dowal, S. L., Puelle, M. R., Nandan, R., \& Inouye, S. K. (2014). NICE to HELP: operationalizing National Institute for Health and Clinical Excellence guidelines to improve clinical practice. J Am Geriatr Soc, 62(4), 754761. doi:10.1111/ jgs. 12768 